Salloum Y. Jodehl A. Thewes M. and König M. (2020). "From forecasting to real-time process controlling with continuous simulation model updates" In: Proc. 37th CIB W78 Information Technology for Construction Conference (CIB W78), São Paulo, Brazil, pp. 440-452. DOI: http://dx.doi.org/10.46421/27066568.37.2020.paper032

\title{
FROM FORECASTING TO REAL-TIME PROCESS CONTROLLING WITH CONTINUOUS SIMULATION MODEL UPDATES
}

\author{
Yara Salloum $^{1}$, Annika Jodehl ${ }^{2}$, Markus Thewes ${ }^{3}$ and Markus König ${ }^{4}$
}

\begin{abstract}
Simulation models become widely used in the industry as they can provide a good prediction of a project's performance to support planning and decisionmaking. Process simulation is particularly interesting in mechanized tunneling projects, where processes interact very sophisticated. The productivity of such projects does not only depend on the performance of the main production processes, but is also significantly influenced by the performance of logistics and maintenance processes. In the planning phase, it is essential to analyze different supply systems and provide a detailed evaluation of them. So far, the simulation models developed are mainly used during the planning phase. However, during the execution of the project many parameters deviate from their predicted values and are subjected to uncertainties and unforeseen events that could not be considered beforehand.

Hence, it is essential to be able to update the simulation model at different stages of the project to get a new prediction of the project performance and to enable an online-steering of the logistics processes. This paper proposes a method to perform a so-called continuous-update of a simulation model, including the validation of the implemented concept to proof that the validity of the online simulation model is maintained.
\end{abstract}

Keywords: process simulation, mechanized tunneling, integration of real-time data, online simulation.

\section{INTRODUCTION}

Tunnel constructions are important infrastructure projects for enhancing transportation networks in congested and fast-growing cities. These projects have usually long durations and large budgets due to their complexity, risks and uncertainties. In general, the conventional methods of tunneling have fewer processes, which make it easier for planning with traditional construction techniques such as preceding diagram method (PDM), critical path method (CPM), program evaluation and review techniques (PERT) or line of balance method (LOB) (Abdalla \& Marzouk, 2013).

This planning process is more complicated in mechanized tunneling projects because of the complexity of the supporting systems of the tunnel boring machine (TBM). The productivity of tunneling projects does not only depend on the TBM performance, for

1 Research Assistant, Ruhr University Bochum, Chair of Computing in Engineering, Bochum, Germany, yara.salloum@rub.de

2 Research Assistant, Ruhr University Bochum, Institute for Tunnelling and Construction Management, Bochum, Germany, annika.jodehl@rub.de

3 Head of Institute, Ruhr University Bochum, Institute for Tunnelling and Construction Management, markus.thewes@rub.de

4 Head of Institute, Ruhr University Bochum, Chair of Computing in Engineering, Bochum, Germany, Bochum, Germany, koenig@inf.bi.rub.de 
example its advance speed, penetration rate or the machine capacity, but subsequently also on the performance of the supporting logistics. These processes, such as the supply chain of the required materials or the transportation systems inside the shaft and the tunnel, can significantly affect the project performance. Any delay or failure in the sequence of processes can delay or stop the tunnel excavation and segmental lining. For the above-mentioned reasons, it is necessary to develop higher sophisticated simulation models, to simulate the interaction and overlapping between these simultaneously operating tasks to predict the progress of the project.

The use of simulation models is currently limited to the planning phase. However, the tendency to use such models as a decision-making tool during the execution phase supports the need to develop dynamic simulation models (Adra, 2016). For this, real-time data needs to be integrated into the model at any stage of the simulation to have a continuous update of the simulation outputs, and an updated prediction of the project performance, especially when unexpected disturbances occur (Law, 2009).

This study presents a concept to update an agent-based simulation model focusing on logistics in a slurry shield tunneling project. This concept is performed in four basic steps to adjust the offline simulation model so that the integration of real-time data at different stages of the simulation is enabled and it conducts suitable validation tests to ensure the validity of the online-simulation model.

\section{BACKGROUND}

In this section, a general overview of the main processes in mechanized tunneling is given and followed by a summarized description of the agent-based model that is used in this study to simulate these processes. A short overview of the development of the online simulation modeling is also included as a background for the presented method for the real-time simulation of the described processes.

\subsection{Processes in mechanized tunneling}

Mechanized tunneling is a highly automated process for the construction of tunnels. With the help of tunnel boring machines (TBM) it is possible to excavate large diameters up to $19 \mathrm{~m}$. For tunnelling in soft ground, mainly shield machines are used. These shield machines are surrounded by a cylindrical steel construction, the shield, which supports the excavated cavity until the final tunnel lining is installed (see Fig.1). In a shield machine the soil is excavated by a large cutting wheel and brought to the surface by pipes or transport vehicles. The installation of the tunnel lining takes place alternately to the excavation of the soil. In the protection of the shield skin, pre-cast concrete segments, the segmental lining, are assembled to a ring and installed as a permanent support of the tunnel cavity (Maidl, et al., 2014). 


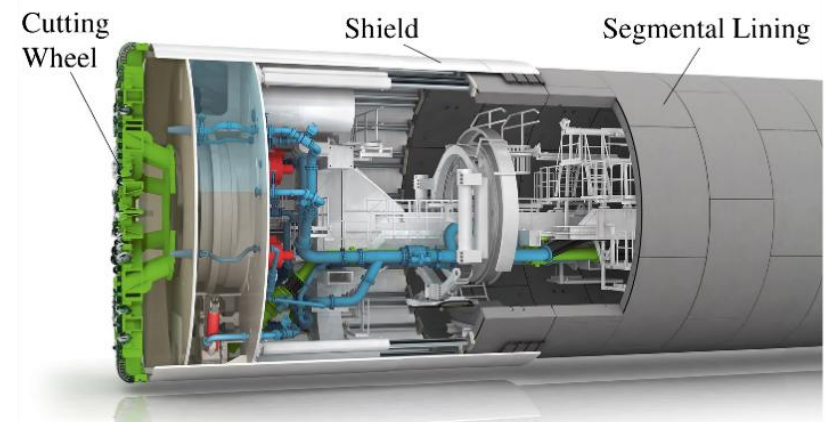

Fig. 1. Construction of a Shield Machine (Mix shield) (Herrenknecht AG)

All types of TBMs have in common, that there are trailing support decks known as the back-up system inside the finished part of the tunnel. The support system located on the back-up includes conveyors or other systems for muck removal, slurry pipelines if applicable, control rooms, electrical systems, dust removal, ventilation and systems for transport of pre-cast segments (Maidl, et al., 2014).

Disturbances in the process flow are unavoidable in the field of mechanized tunneling, but it is necessary to identify sources of interference and minimize them wherever possible. Disturbances occur both in the area of propulsion and in the area of logistics. Often, downtimes caused by failures are representing more than $40 \%$ of the total project time (Duhme, 2018).

Rahm (2017) defines seven possible causes for disturbances in mechanized tunneling. These include:

- Difficult geological conditions

- Extension of supply lines, pipes, and tracks

- Maintenance work

- Technical failures

- Inefficient, congested or sensitive logistics processes

- Modification of the machine and logistics elements

- Exceptional events

\subsection{Description of the Simulation model}

Simulation models are composed of elements that display the elements of a real system. Depending on the subject to be analyzed, the systems are simplified. For this research, an agent-based simulation model for a mechanized tunneling project including the logistics operations is used. The model is implemented in the software Anylogic (The Anylogic Company, 2019), which is a java-based multi-method simulation framework, which uses Discrete Event Simulation.

The proposed concept to update simulation models in this study is applied to this model. The model consists of 22 agents. Each agent presents one element of the TBM and the jobsite logistics. Figure 2 illustrates the block definition diagram of this agentbased model. 


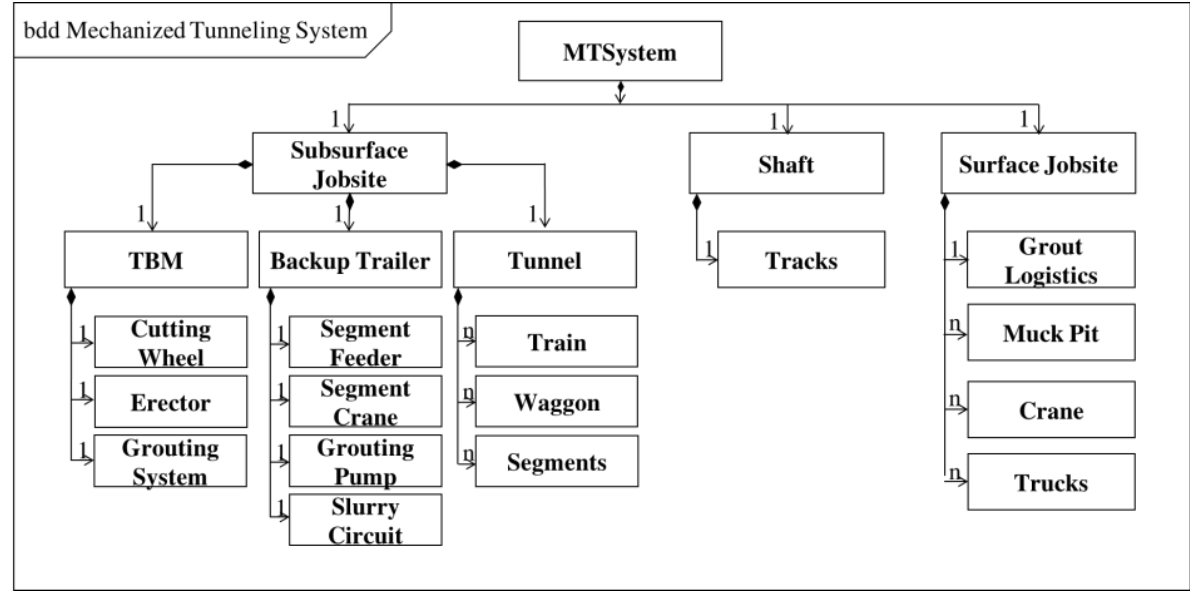

Fig. 2. Block definition diagram of the studied mechanized tunneling simulation model

The three agents of the first level represent the three main areas of a mechanized tunneling project (the subsurface jobsite, the shaft and the surface jobsite). The agents in the second level represent the processes taking place in each area. Thus, the cutting wheel, the erector and the grouting system are the main elements of a TBM.

Agents operate in a discrete manner, but they interact with each other through signals and messages to communicate and mimic the interaction of the real system. In addition, each agent contains of various parameters, variables and statecharts. Parameters describe the main properties of the simulated process, such as capacities and properties of the equipment and machinery or initial flow rates of pumps. Statecharts describe the state of an element and define the conditions under which a state is changed. The variables, however, are used to store the state of an agent.

\subsection{Real-time update of Agent-based Simulation models}

The evolution of simulation tools has been driven by the rapid evolution of computing technologies. As computing technologies have decreased in cost and increased in performance, the capability of simulation tools to solve increasingly complex problems in less time has improved.

In the last few years a lot more studies followed, which aimed to develop simulation models for mechanized tunneling projects (e.g. AbouRizk et al. (2011)). First models were developed in CAD and 3D modeling software mainly to simulate the boring process and the cutting wheel. Kasper et al. (2004) presented a 3D finite element model of shield-driven tunnel excavation in soft ground. This model considers the main factors that control and affect the excavation process such as the soil and the ground water, the frictional contact of the boring machine to the soil, the hydraulic jacks, the tunnel lining and the tail void grouting.

Liu et al. (2010) presented another simulation model for hard rock TBM using the CYCLONE framework. The processes are modeled on a rather abstract level but involve interactions between on-site logistics and production processes by taking into account the disposal of muck. The authors identify a suitable logistic setup through variation of three parameters: muck cars per train, number of trains and rail infrastructure. Until then most models are isolated and presented on one level of the process without taking into consideration the influence of other processes in different layers of the project such as supporting logistics operations and maintenance. 
Rahm et al. (2013) suggested an approach to simulate the TBM machines and the progressing rate of the machine, taking into consideration the disturbances and investigating the effect of technical failures related to the process. A jobsite logistics simulation was presented by Scheffer et al. (2014), which focuses on the supply chain of a tunneling project. This model connects the operations on surface with the subsurface processes and allows interactions in between.

As noticed in the tunneling projects, a high percentage of delays in project's execution occur due to delays and disorder in logistics and supply chains. Because of that, a lot of studies were more focused on the simulation of logistics and maintenance. In 2017 Rahm investigated disturbances in the logistic chain of mechanized tunneling projects with the help of process simulation (Rahm, 2017). In 2018, Duhme (2018) analyzed the simulation based planning of logistics processes in mechanized tunneling. Besides, Conrads et al. (2018) investigated a detailed simulation of the maintenance strategies considering various wear prediction models for cutting tools in order to suggest an optimal maintenance plan for the TBM.

Simulation models developed in these researches contain many non-deterministic variables (i.e. the variables that present the frequency of the failure's occurrence in the operating equipment and the duration of required maintenance). These uncertainties are usually displayed by distribution functions.

The recent studies of process simulation modeling of construction and production systems suggest that models should be data-driven during the simulation. Data-driven simulations are common in mathematical models but their applications to agent-based models are not technically mature yet. However, the integration of real-time data into simulation models enables the possibility of comparing simulations to the real system during the project's execution. The incorporation of real-time data into agent-based models improves the predictive ability of such models and results in increasingly wellcalibrated model parameters and more accurate outcomes (Oloo \& Wallentin, 2017).

The most essential step to manage the online-update of the simulation system is the effective management of the simulation inputs, which means the ability to update the inputs of the model at any time. In agent-based models, each agent operates in a discrete manner, using the parameters and variables implemented in the agent. The initial values of these variables are defined at the beginning of the simulation run and the simulation starts to operate from this state to the specified endpoint sequentially in a state based order until a break- or end-state is reached. It is important to ensure that the interaction between the agents is not disturbed by the change of the initial state of the variables. However, the main challenge in the update process is to maintain the validity of the model after the update in order to ensure that the model is still working properly after the new implementation. Although the agents are isolated during execution, to implement the update, extra variables or time-related functions need to be added, which may require a validation step afterwards.

\section{METHOdology}

Following, a method to update a computer agent-based simulation model to predict the overall project duration and the interaction between the different production, logistics and maintenance tasks in TBM tunneling projects is presented. The main concept of the online update of a simulation model can be summarized in four basic steps, which are illustrated in Figure 3. 
Methodology for the online update of a simulation model

\begin{tabular}{l} 
Step 1 \\
\hline $\begin{array}{l}\text { Choose Inputs to } \\
\text { Update }\end{array}$ \\
\hline Real System Inputs \\
\hline $\begin{array}{l}\text { Simulation Internal } \\
\text { Inputs }\end{array}$ \\
\hline
\end{tabular}

\begin{tabular}{|l|}
\hline Step 2 \\
\hline $\begin{array}{l}\text { Integrate Real-Time } \\
\text { Data }\end{array}$ \\
\hline \\
$\quad \begin{array}{l}\text { Nondeterministic } \\
\text { Inputs }\end{array}$ \\
Deterministic Inputs
\end{tabular}

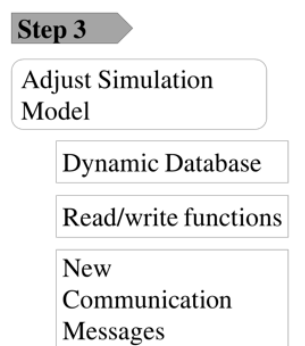

Step 4

Validation Test

Fixed Input Values

Fixed probability

Functions

Fig. 3. The methodology suggested to update an ABM simulation model Implementation

First, the main input parameters must be identified that affect the objective of the simulation the most (step 1). These input parameters can be classified into two main types. The first type are the real-system inputs, such as the specifications and capacities of the equipment. The second type are the internal simulation inputs, which have been added by the designer to store values during the simulation and to regulate the flow of the processes during the execution of the simulation. The second step is to integrate the real-time data in the simulation model (step 2). In TBM simulation models, a large number of inputs have a non-deterministic nature, for example, they don't have a fixed value but they are randomly predicted by mean of probability distribution functions (PDFs). For the deterministic inputs, the integration in the model's database is simple and direct. In contrast, the integration of non-deterministic inputs requires extra analysis and comparison between the real-time data and the data gatherd from similar previous projects to update the predefined probability distribution functions.

If the model is designed for offline simulation during the planning phase, some modifications might be necessary for the next step to adapt the real-time data for an online update of the simulation model (step 3). The first adjustment is to create a dynamic database for the inputs of the process. The input's database contains the initial values and states of the elements variables. Other modifications might be also necessary to collect and insert data at different stages of the model execution. The last step includes the validation of the implemented concept, taking into consideration the nondeterministic nature of the simulation variables, to ensure the credibility of the modified model (step 4).

This process can turn the offline simulation model into a useful tool to support the decision-making process during the execution of the project.

\subsection{Identifying the essential input parameters for the online-update}

Since the focus of this study is on the overall project duration and the performance of the logistic processes on the construction site, the simulation parameters and variables that control these processes must be investigated.

The project duration is related to the core processes of excavation, ringbuild and the performance of the supporting processes. On top of that, the duration of the project's downtime is related to the technical failures of each process and the subsequent delay in the logistics supplies. To monitor these variables in each agent, their initial values are connected to the input database. Figure 4 illustrates the main five agents in the simulation model and a sample of the connected variables to the database. 


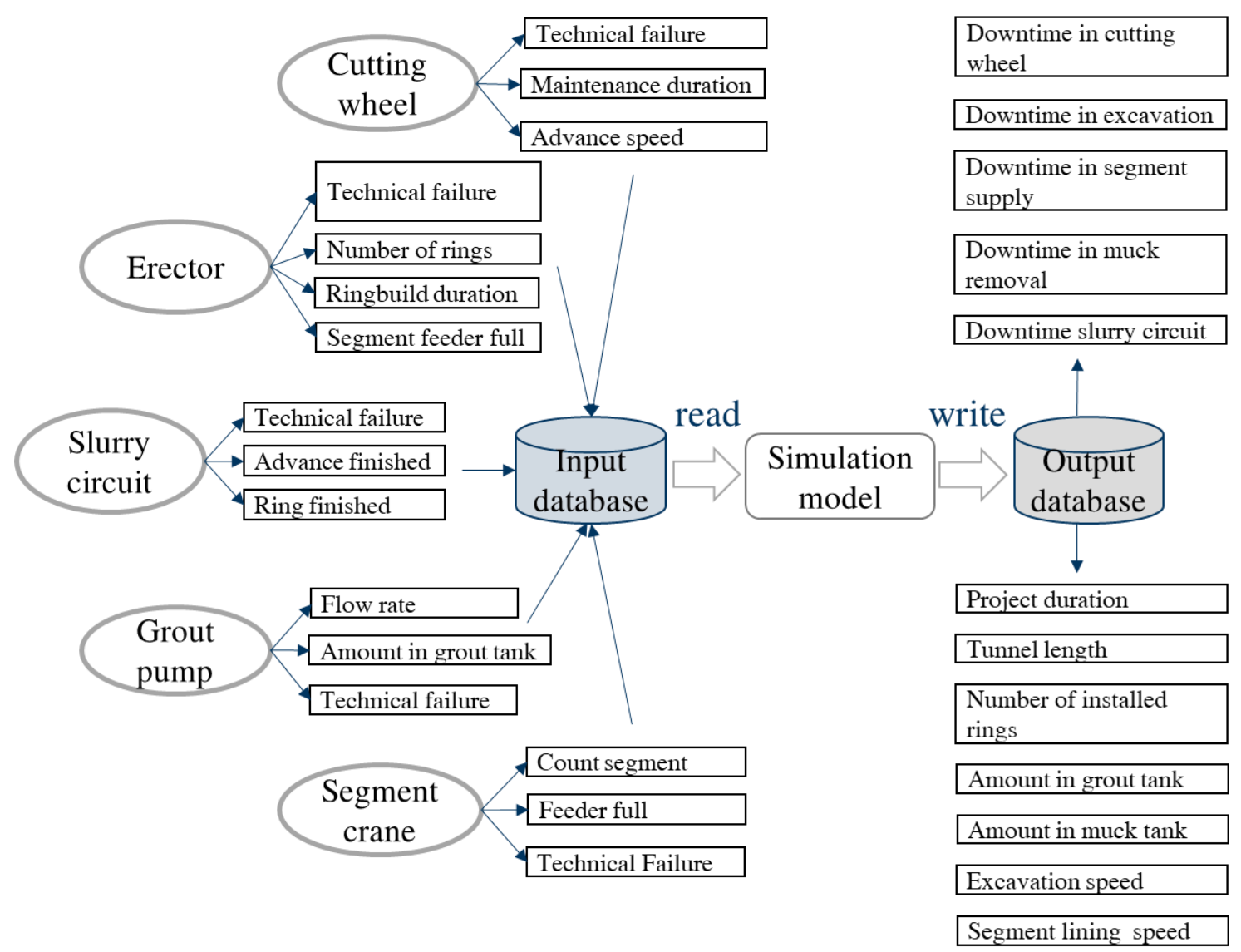

Fig. 4. Sample of the inputs and outputs connected to the simulation databases for the update phase

The simulation model will read the initial values at the beginning of the run. Nevertheless, the current values of the output variables are monitored during the simulation and will be stored in the output database file at the end of the simulation or at any desired stage of the project. The outputs of the simulation are mainly the duration of the project, the duration of the excavation and the duration of downtimes. In order to perform an actual target comparison in the course of the update, more data should be collected, such as the current status of the tanks, the current advancing speed, the current average process durations, the number of installed rings, and the current length of the tunnel.

These output values are compared with the real-time values at different stages of the simulation to detect any deviation. Accordingly, the input database can be updated with real-time data and the simulation can be executed again to get the updated results to predict the performance of the project for the next phase.

\subsection{Integration of real-time data}

The integration of real-time data can be tricky because of the non-deterministic nature of some variables. In the proposed TBM simulation model, different types of probability distribution functions (PDFs) are set in the planning phase to predict certain values. This could be, for example, the occurrence of technical failures in the system, the duration of failures and the advancing speed of the cutting wheel through different types of soils.

In the update phase, direct integration of deterministic input parameters in the model's input database will be performed. The current status of the processes should also 
be updated. In addition, the current length of the tunnel is updated to recalculate the paths of the transportation wagons inside the tunnel. To update non-deterministic data, a different approach will be used. Figure 5 shows the suggested concept to update the PDFs used to evaluate the probability of certain parameters offline (Brooks-Bartlett, 2018).

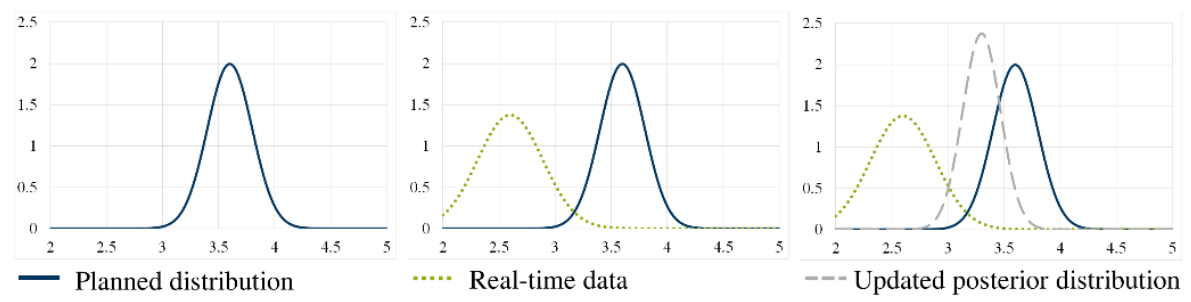

Fig. 5. Update approach for the probability distribution function

In this figure, the blue curve displays the PDF used in planning, which has been generated with a probabilistic approach using data from similar previous projects. During the execution of the project a new set of data, the real-time data, is now available. At a certain point in time, this data is gathered and analyzed to evaluate the validity of the proposed PDF. The green curve represents the probability function of the actual realtime data. Considering both sets of data, we can predict an updated posterior PDF (grey curve) that is more realistic and gives a better prediction of the project performance in the next phase of execution. This is one suggested way to consider real time data within the update without neglecting historical data, as historical data may still be relevant for the further prognosis of a project.

\subsection{Modification of the offline simulation model}

Figure 6 displays the main four steps to prepare offline simulation models for implementing an update. For the update phase, the initial values of the input/output parameters need to be dynamically changeable during the online-simulation.

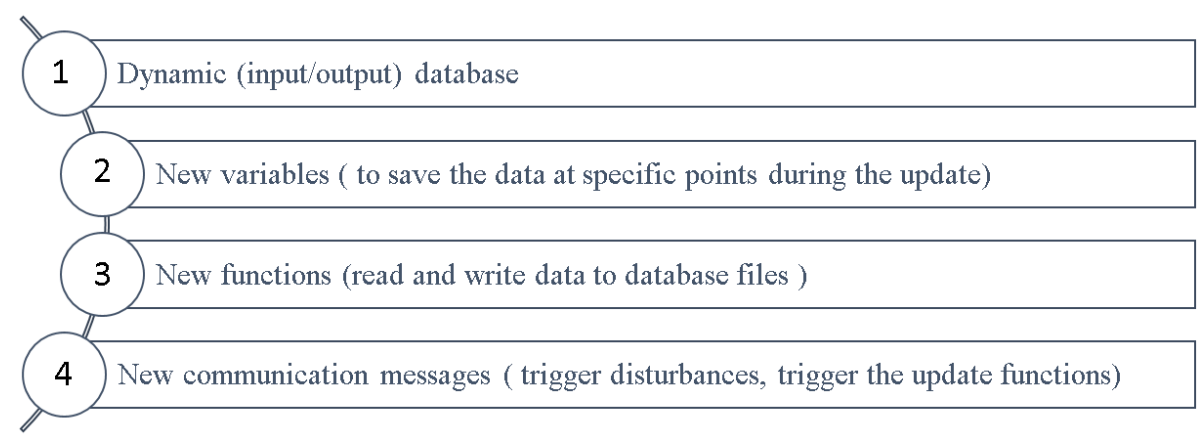

Fig. 6. The main steps to adjust the offline agent-based TBM model to implement real-time data

In the second step, new variables might be added to adjust the offline model in order to store the values of some variables dynamically during the execution of the simulation, which is needed for the evaluation of the current state in comparison with the real-time data. The third step is to create extra read/write functions to the model's agents to $\mathrm{read} /$ write inputs at different points during the execution of the simulation. The same concept is used to register the simulation outcomes before and after the update. The fourth step is to add the necessary communication messages between the agents to 
trigger the read/write functions at the desired stage of the simulation, or to trigger the unexpected disturbances that caused the deviation in the project's performance.

\subsection{The validation of the implemented update concept}

Validation tests are necessary to check the credibility of a model. The offline simulation model used in this research has been validated beforehand, so only an explicit validation of the update method itself is necessary. To ensure the validation of the simulation model, four tests have been conducted. One validation was conducted with a fixed value test. This validation will be shown exemplary in detail in this paper. Besides of this test, a test with fixed seeds (PDF), a test with various seeds and a test with Monte Carlo simulation has been conducted for the validation. These tests have shown that the simulation model is still valid after the implementation of the update method.

Figure 7 displays the basic steps of the validation concept developed for this study.

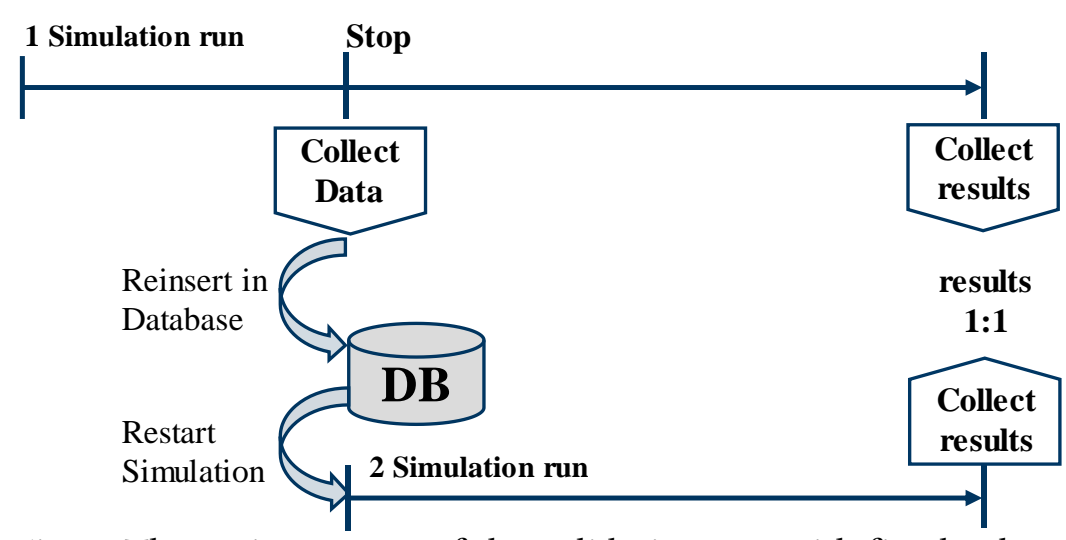

Fig. 7. The main concept of the validation test with fixed values

This validation concept is performed for the fixed value test. For this, the probability of various parameters in the simulation model is eliminated by setting the initial values of all variables to fixed values during the simulation run. The first run of the simulation from the initial point to the end gives the initial prediction of the project duration and performance. For the validation, the concept suggests to create synthetic validation data by stopping the simulation at one stage (for example after building 400 rings of the total 1000 rings) and then collect the output parameters at this point. Subsequently, the synthetic data is reinserted in the input database of the model and the updated simulation starts again from this new initial point to obtain the simulation results. Then, the results from the updated to the original simulation run are compared.

Therefore, the results shown in Table 1 must be hypothetically identical. A small deviation is detected though, due to the fact that some events in the model are controlled with timers during the simulation, which are restarted and then are causing time overlapping. Furthermore, some deviation is caused by the lack of tracking the moving parts as transportation vehicles, which is simplified in this model and which cannot be located during the simulation, resulting in the assumption to start from their initial position every time the simulation is executed. 
Table 1. The results of the first validation test of the update concept

\begin{tabular}{lcccc}
\hline & & $\begin{array}{c}\text { Updated } \\
\text { Simulation Model }\end{array}$ & $\begin{array}{c}\text { Original } \\
\text { Simulation Model }\end{array}$ & deviation \\
\hline Project duration & [day] & 145.50 & 145.73 & $-0.16 \%$ \\
Excavation advance & [hours] & 518.00 & 518.00 & $0.00 \%$ \\
Ring building & [hours] & 760.68 & 761.03 & $-0.05 \%$ \\
Failure cutting wheel & [hours] & 51.67 & 51.67 & $0.00 \%$ \\
Technical failure grout pump [hours] & 41.00 & 41.00 & $0.00 \%$ \\
Failure crane & [hours] & 0.50 & 0.50 & $0.00 \%$ \\
Maintenance erector & [hours] & 96.00 & 96.00 & $0.00 \%$ \\
\hline
\end{tabular}

\section{CASE STUDY}

In this case study, the online-update method of the simulation model is tested as a planning and management tool during the execution of the project. Figure 8 illustrates the concept of the implementation taking the nondeterministic nature of the variables into consideration.

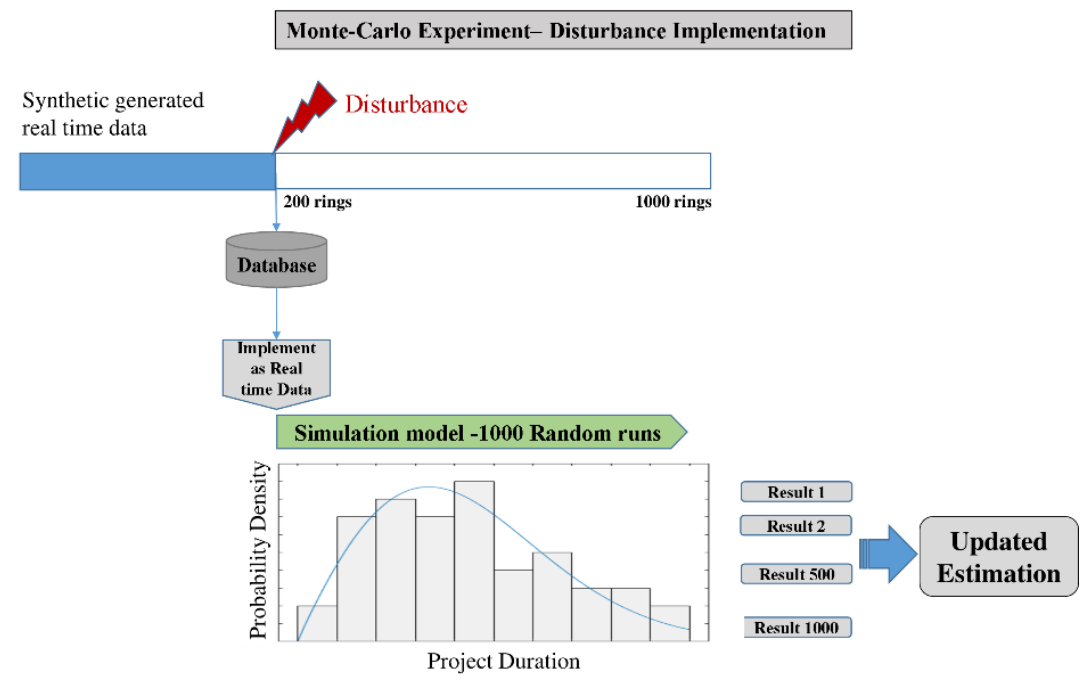

Fig. 8. Developed concept for the update of simulation models (case study)

In this case study, real time data is synthetically and exemplary generated. For the generation of the real time data, a simulation run is performed until the lining of 200 rings. At this stage, an unpredicted disturbance is simulated, which is assumed as a failure in the cutting wheel for 200 hours (i.e. 8.33 days). After the disturbance passes, the simulation output data is generated and used as the real time data. For the update of the projects performance, these real time data will be reinserted in the models' database as the initial value for the new run of the simulation. Due to the nondeterministic nature of the model, a Monte-Carlo experiment of 1000 runs will be performed from this point to the end of the project to get a new prediction of the project's duration and performance after the occurrence of this unplanned downtime of the cutting wheel. 


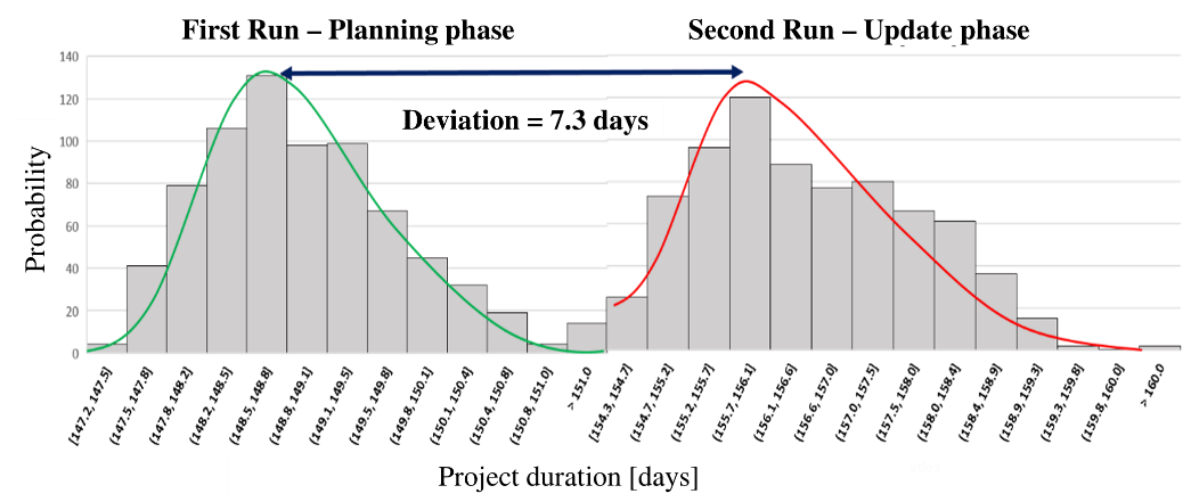

Fig. 9. Project Duration curves in the planning phase and update phase

Figure 9 shows the comparison between the initial results of the simulation in the planning phase and the results of the updated model after the occurrence of the extra failure during the execution of the project.

As shown, the most frequent prediction of the project duration in the initial case is 148.65 days, and for the updated simulation it is 155.9 days. The maximum predicted duration in the initial case is about 151 days. This value has increased to 160 after the disturbance. The deviation between the minimum and maximum value of the project duration in the initial case is about 4 days and it has increased to 6 days after the implementation of the disturbance.

Accordingly, an offset between the two curves can be noticed and it is rational regarding the implemented error. The 200 hours delay in the first phase of the excavation (i.e. 8.33 days) caused a total delay of 7.3 days in the total prediction of the project duration. This delay cannot exactly be the same at the end of the simulation because of the complex interaction between the agents (i.e. the processes in the real system). The malfunction of the cutting wheel for a certain time can affect the following processes. For example, it causes the erector to stop. Nevertheless, it may not affect the supply chain of the segments. Hence, the project duration is expected to be extended accordingly. However, during the maintenance of the cutting wheel, other preventive maintenance tasks can be executed and the delay in other tasks can be compensated. This is only a small example of the interaction that is analyzed in the simulation model. The real case can be more complicated, when more than one unplanned failure occurs, which might affect the project dramatically or has no significant effect at all. This analysis of the situation cannot be done without a proper online-simulation model.

\section{CONCLUSION}

Simulation models can be a useful managing and decision-making tool during the progress of a project. This can be achieved by developing online-simulation models that allow the adaption of real-time data at any stage of the project execution when the project's outcome starts to deviate from the predicted results. The integration of the realtime data in the simulation allows to obtain updated outcomes, which can help to take countermeasures when unplanned disturbances occur along the execution of the project. This paper proposed a method to turn an offline agent-based simulation model for TBM jobsite logistics into an online model to update the prediction of the project's duration and performance. In particular, if unplanned disturbances occur, this allows to evaluate the effect of these disturbances and to take suitable steps to optimize some processes or 
recover the downtime in the project's execution. This update is performed in four essential steps and validated afterwards. In the end, a practical example of the use of the online-simulation model is presented to explain the main purpose of the suggested concept.

In following research, it is planned to use this method to update simulation models with adapted non-deterministic data and to investigate their effects on the system.

\section{ACKNOWLEDGMENTS}

The authors would like to acknowledge the financial support for this research received from the German Science Foundation (DFG) within the framework of the subproject C3 of the Collaborative Research Center SFB 837.

\section{REFERENCES}

Abdalla, M. and Marzouk, M. (2013). Planning of Tunneling Projects using Computer Simulation and Fuzzy Decision Making. Journal of Civil Engineering and Management, p. 18.

Adra, H. (2016). Real-time predictive and prescriptive analytics with real-time data and simulation. In: Proceedings of the 2016 Winter Simulation Conference. Crystal Gateway Marriott, Arlington, USA, p. 6.

AnyLogic 8 University 8.4.0. (2019). The Anylogic Company.

Brooks-Bartlett, J. (2018). Probability concepts explained: Bayesian inference for parameter estimation. [online] towardsdatascience.com. Available at: https://towardsdatascience.com/probability-concepts-explained-bayesian-inferencefor-parameter-estimation-90e8930e5348 [Accessed 2019].

Conrads, A., Scheffer, M., König, M. and Thewes, M. (2018). Robustness Evaluation of Cutting Tool Maintenance Planning for Soft Ground Tunneling Projects. Underground Space, 3(1), pp. 72-85.

Duhme, R. (2018). Deterministic and Simulation Based Planning Approaches for Advance and Logistic Processes in Mechanized Tunneling. Bochum: Ruhr-Universität Bochum, Universitätsbibliothek.

Ebrahimy, Y., AbouRizk, S., Fernando, S. and Mohamed, Y. (2011). Simphony Supply Chain Simulator: a simulation toolkit to model the supply chain of construction projects. Simulation, Volume 87, pp. 657 - 667.

Herrenknecht AG. Mixshield - Herrenknecht AG [online] www.herrenknecht.com. Available at: https://www.herrenknecht.com/en/products/productdetail/mixshield/ [Accessed 10 Jan. 2020].

Kasper, T. and Meschke, G. (2004). A 3D finite element simulation model for TBM tunnelling in soft ground. International Journal for Numerical and Analytical Methods in Geomechanics, p. 16.

Law, A. M. (2009). How to build valid and credible simulation models. In: Proceedings of the 2009 Winter Simulation Conference. Tucson, USA, p. 10.

Liu, D., Zhou, Y. and Jiao, K. (2010). TBM construction process simulation and performance optimization. Transactions of Tianjin University, 16(2010), p. 194-202.

Maidl, B., Thewes, M. and Maidl, U. (2014). Basics and Aditional Services for Design and Construction. In: Handbook of Tunnel Engineering. Berlin: Ernst \& Sohn Verlag, p. 458. 
Oloo, F. and Wallentin, G. (2017). An Adaptive Agent-Based Model of Homing Pigeons: A Genetic Algorithm Approach. IJGI, p. 12.

Rahm, T., Sadri, K., Koch, C., Thewes, M., König, M. (2013). Evaluation of disturbances in mechanized tunneling by using multi-method simulation. Journal of Computing in Civil Engineering, ASCE.

Rahm, T. (2017). Simulation-based Evaluation of Disturbances of Production and Logistic Processes in Mechanized Tunneling Operations. Bochum: Ruhr-Universität Bochum, Universitätsbibliothek.

Scheffer, M., Rahm, T., Duhme, R., Thewes, M., König, M. (2014). Jobsite Logistics Simulation In Mechanized Tunneling. In: Proceedings of the 2014 Winter Simulation Conference. Bochum. 